\title{
FIBROSE PULMONAR POST-GRIPPAL
}

\author{
(Contribuição ao seu estudo.) \\ Por Antonio Bernardes de Oliveira
}

Trabalho do Instituto Anatomo Pathologico da Faculdade de Medicina e Cirurgia de S. Paulo.

O assumpto da nossa communicação apresenta um certo interesse por se tratar de uma forma pathologica individualisada em um quadro clinico mais ou menos bem definido, e pela certa actualidade que a epidemia de grippe trouxe ao mesmo.

Já na epidemia de 1837 , Graves chamara a attenção para a difficil distinção entre a peneumonia grippal chronica e a tuberculose dos pulmões.

Teissier, Chatin e Collet descreveram um grupo de casos com "forma pseudo-phymica".

Kundrat e Weichselbaum trataram da pneumonia chronica intersticial indurativa.

Modernamente, varios trabalhos têm apparecido sobre o assumpto, principalmente graças á contribuição de autores americanos, como MacCallum e outros.

Um certo descuido por parte do $_{S}$ autores em descrever e estudar a fibrose do pulmão não especifica encontra sua explicação na raridade dessas eventualidades após as pneumonias communs.

A pneumonia grippal apresenta, porém, o caracter especial de produzir muito frequentemente essa lesão.

A documentação photographica é toda original e proveniente de material de nossas autopsias.

\section{Anatomia pathologica.}

Os pulmões tomados pela broncho-pneumonia fibrosante postgrippal apresentam esclerose de extensão variavel. Quando não se produz um exito lethal, essa fibrose evolue, tornando-se estão apreciavel a olho nú.

A tendencia para a proliferação fibrosa é tal que muito precocemente e em pleno periodo agudo já é visivel ao microscopio.

Raro é, porém, que as condições locaes pulmonares permittam uma sobrevivencia bastante longa para que se produza uma fíbrose tão extensa como a do nosso primeiro caso. Fig. 1 . 


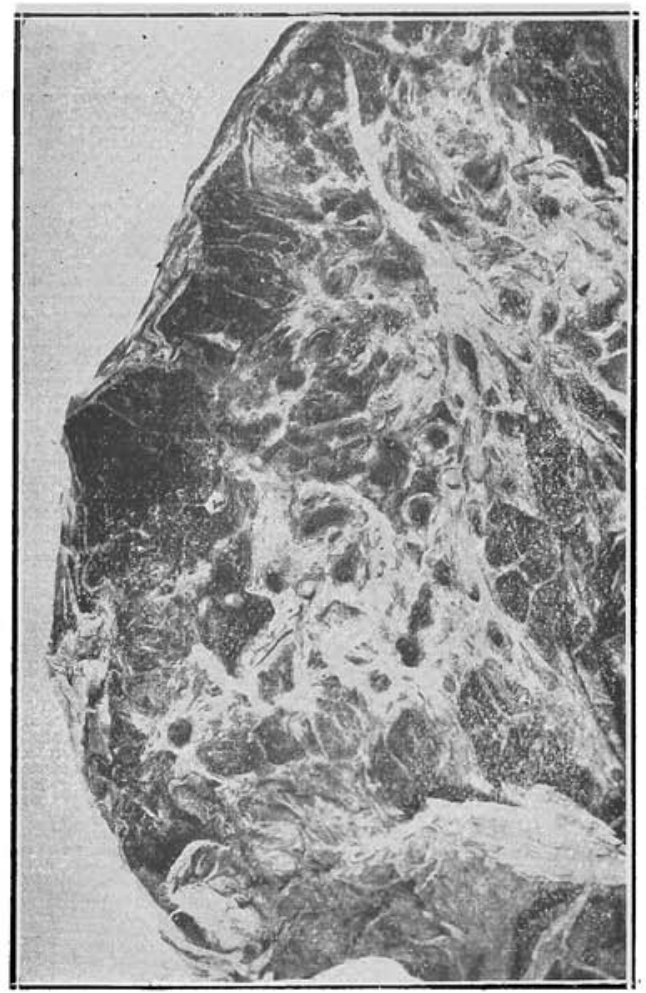

FIG. 1

Extensa fibrose pulmonar post-grippal 


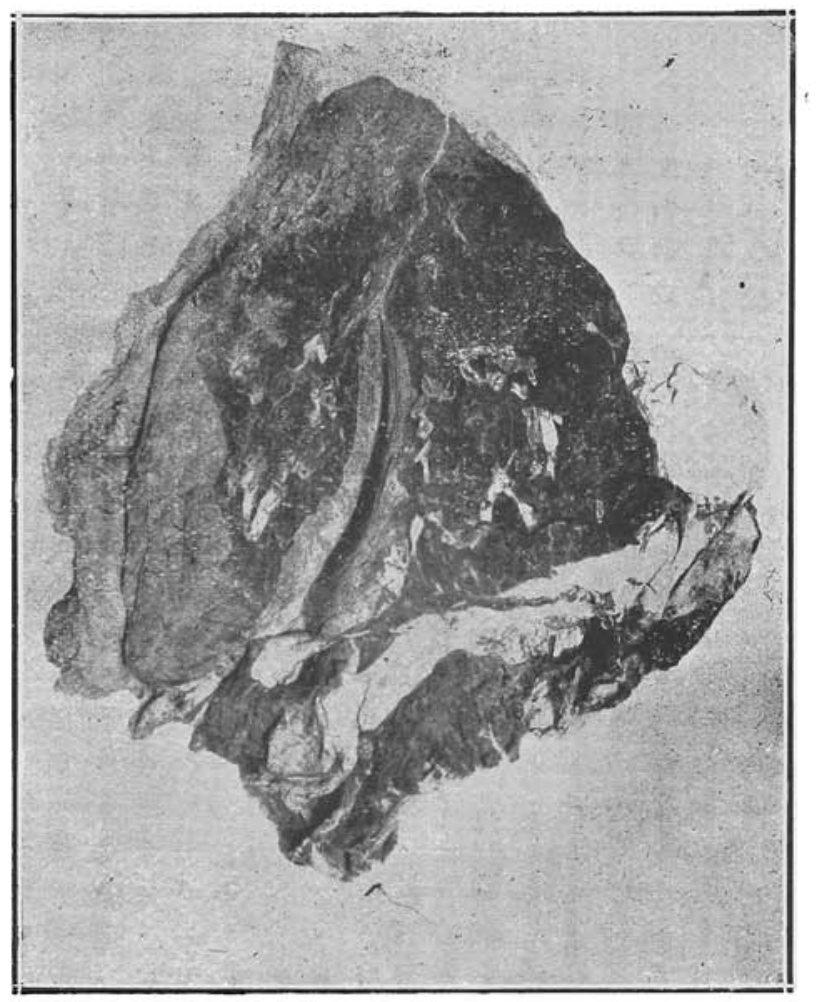

FIG. 2

Fibrose tuberculosa do apice pulmonar 


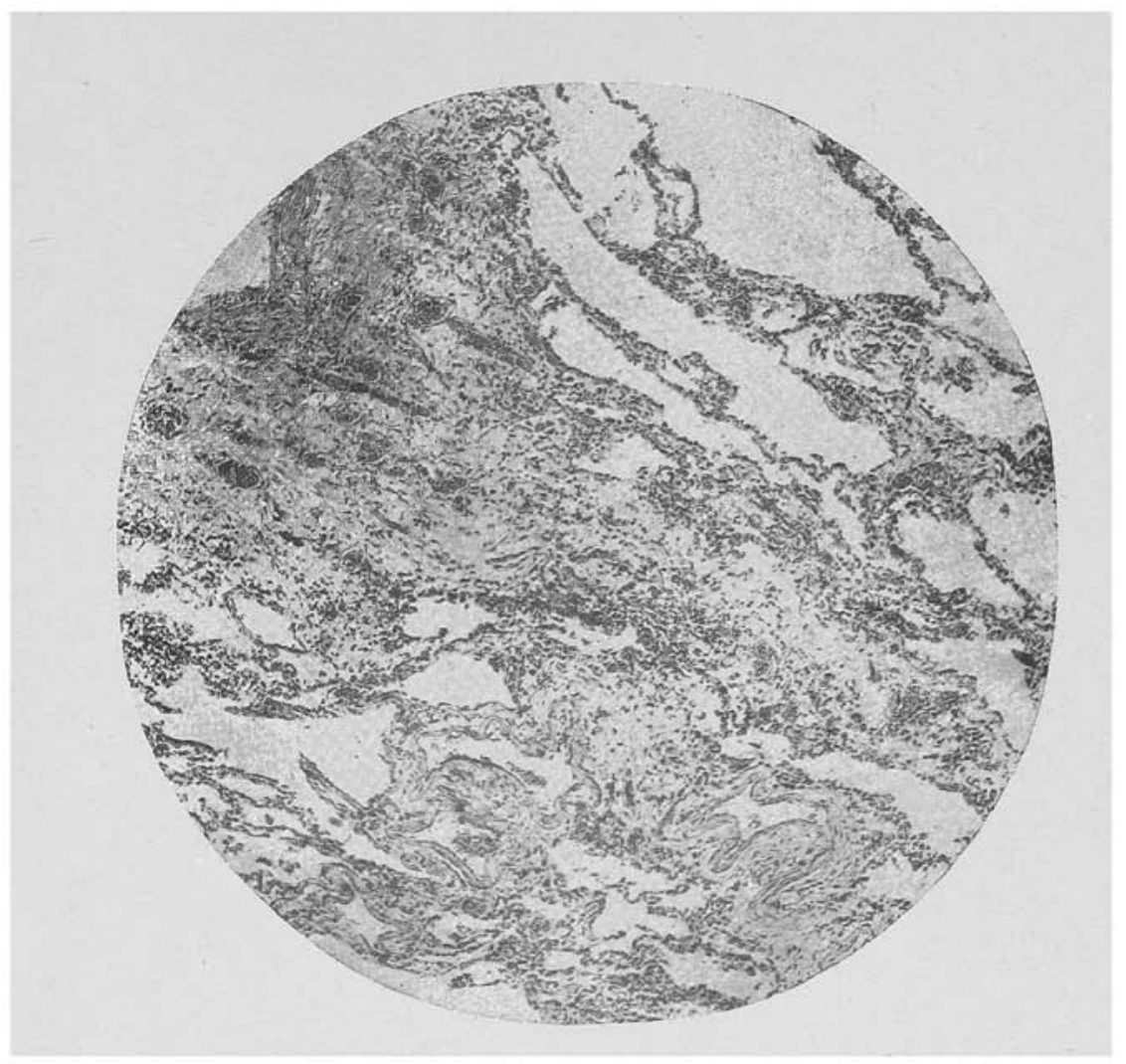

FIG. 3

Faixas fibrosas peri-vasculares. Emphysema compensatorio. 


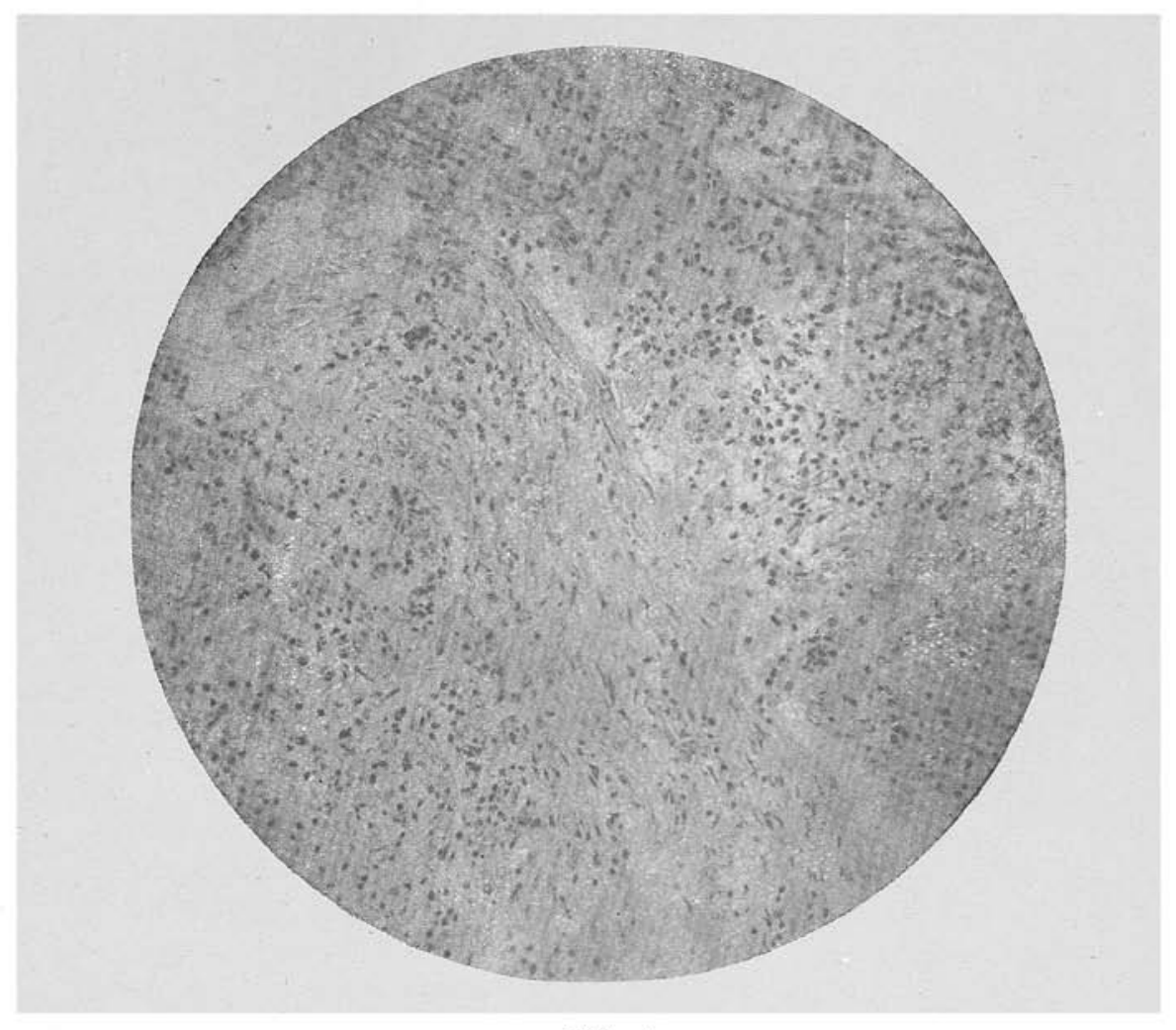

FIG. 4

Bronchiolite obliterante 
Geralmente, nas autopsias a esclerose é mulito menos intensa, e mascarada pelos processos inflammatorios agudos.

O orgão apresenta faixas conjunctivas cicatriciaes dispostas ao longo dos bronchios e vasos, percorrendo o parenchyma pulmonar de modo a delimitar areas maiores ou menores de tecido pulmonar mais conservado. Partindo dessas faixas maiores, vêm-se ramificações mais delgadas. Não são visiveis areas calcificadas nem necrosadas.

Os principaes caracteristicos das lesões são: a bilateralidade, a extensão, a localisação peri-bronchica, a precocidade e a ausencia de vestigios de tuberculos e gommas.

Os bronchios acham-se muito espessados e mostram-se proeminentes, apresentando muito frequentemente dilatações (bronchiectasias).

A superficie pleural quasi constantemente está tomada por um processo pleuritico chronico que se traduz por adherencias firmes e espessamentos accentuades na pleura diaphragmatica. (Fig. 1).

Esse tecido conjunctivo individualisa a fibrose post-grippal, distinguindo-a das tuberculoses fibrosadas e formas esclerosantes da syphilis pulmonar.

A fibrose tuberculosa tem localisação frequente no apice, ê mais delimitada, interessa mais ou menos homogeneamente toda uma area e mostra vestigios microscopicos do processo que a originou, o tuberculo. (Fig. 2).

Differencia-se da fibrosa syphilitica pela extensão, bilateralidade, ausencia de vestigios de gommas, assim como de outras lesões organicas syphiliticas.

10 estudo microscopico e analytico nos mostra essas areas fibrosas dispostas ao longo dos vasos e bronchios. (Fig. 3).

As paredes dos bronchios acham-se muito espessadas e constituidas por um tecido conjunctivo cicatricial encerrando grande numero de vasos dilatados cheios de sangue.

A mucosa bronchica está descamada em grandes extensões, deixando á mostra um tecido de granulação com reação inflammatoria chronica.

NNo seio do tecido conjunctivo vêmos alguns bronchiolos com vestigios de proliferação epithelial e no seu interior exsudato inflammatorio em via de organização. Fig. 4.

Esse processo foi pelos autores americanos denominado de bronchiolite obliterante.

Interessante é notar que aqui, como na cirrhose hepatica, ha uma tentativa de regeneração por parte do epithelio dos bronchiolos que traduz por figuras canaliculares no seio do tecido conjunctivo. 
Esses vestigios bronchicos são envoltos por um manguito inflammatorio chronico. E' possivel reconhecer os alveolos fibrosados na massa conjunctiva. (Fig. 5).

A broncho-pneumonia grippal tem especial predileç̧ão para os lymphaticos pulmonares que são tomados por uma lymphangite obliterante. (Fig. 6).

Segundo alguns, seria essa obliteração precoce e definitiva doe lymphaticos a principal causa da caracteristica tendencia fibrosante desse typo de broncho-pneumonia, pois esse obice á circulação lymphatica diffficultaria a remoção dos exsudatos inflammatorios dos bronchiolos e alveolos, permittindo assim a sua gradual organisação.

O processo de fibrose se realiza por uma bronchiolite e alveolite fibrosantes.

O restante parenchyma pulmonar apresenta em muitos pontos emphysema e um certo grau de congestão.

Não são visiveis areas calcificadas nem de necrose caseosa ou gommosa.

Quanto á significação dessa fibrose, consideramol-a como um processo de reparação cicatricial aos damnos causados pelo alto poder necrosante dos germens infectantes.

Desde que a lesão seja tal que torne impossivel um restitutio ad integrum, a reparação far-se-á pela substituição fibrosa.

Uma fibrose inicial do mesmo typo é possivel realisar-se experimentalmente em animaes pela inhalação de gazes de guerra, como fizeram meu estimado mestre, prof. Lambert, e outros.

Essas experimentações evidenciam a influencia da necrose severa na genese da fibrose.

Com o intuito de avaliar a frequencia da fibrose post-grippal, organizei uma estatistica, utilisando-me de 624 protocollos de autopsias realisadas na Escola de Medicina de Universidade de Yale, no periodo de 1818 a 1922 , protocollos esses fornecidos pelo prof. Lambert.

Escolhi dentre elles os casos de broncho-pneumonia grippal, asignalando a frequencia relativa das formas fibrosantes. Todos esses casos foram cuidadosamente estudados macro e microscopicamente, e quasi todos têm| exame bacteriologico que denuncia a frequencia do B. de Pfeiffer, Streptococcus hemolyticus, Pneumococcus de varios typos e outros em menor proporção.

Dos 151 casos de influenza, 41 mostravam fibrose em maior grau, o que dá uma frequencia de $27,1 \%$.

o quadro junto, eschematisado pelo diagramma, representa os nossos resultados. (Fig. 7). 


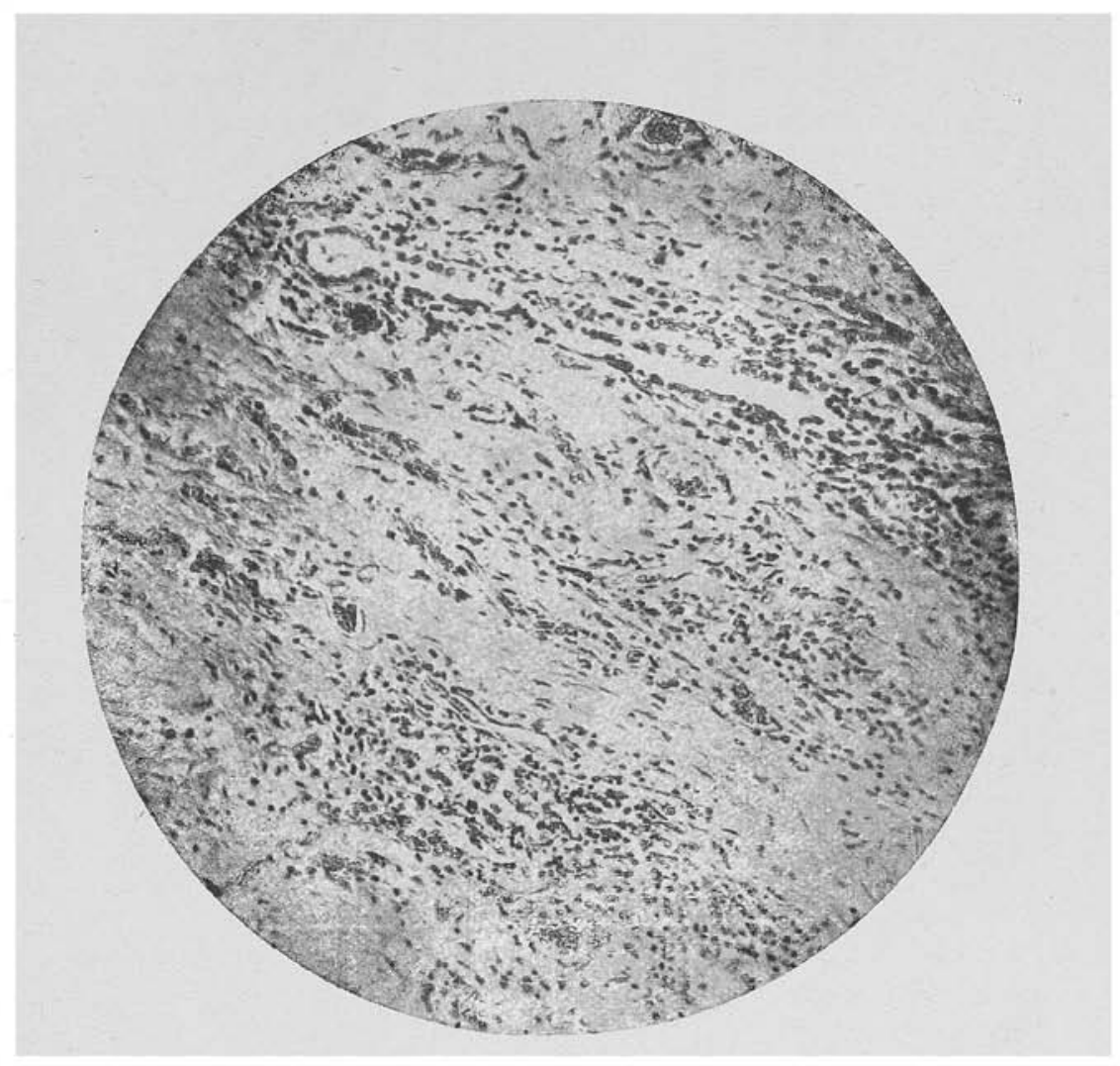

FIG. 5

Figura canalicular na massa conjunctiva. Em baixo, dois alveolos fibresados 


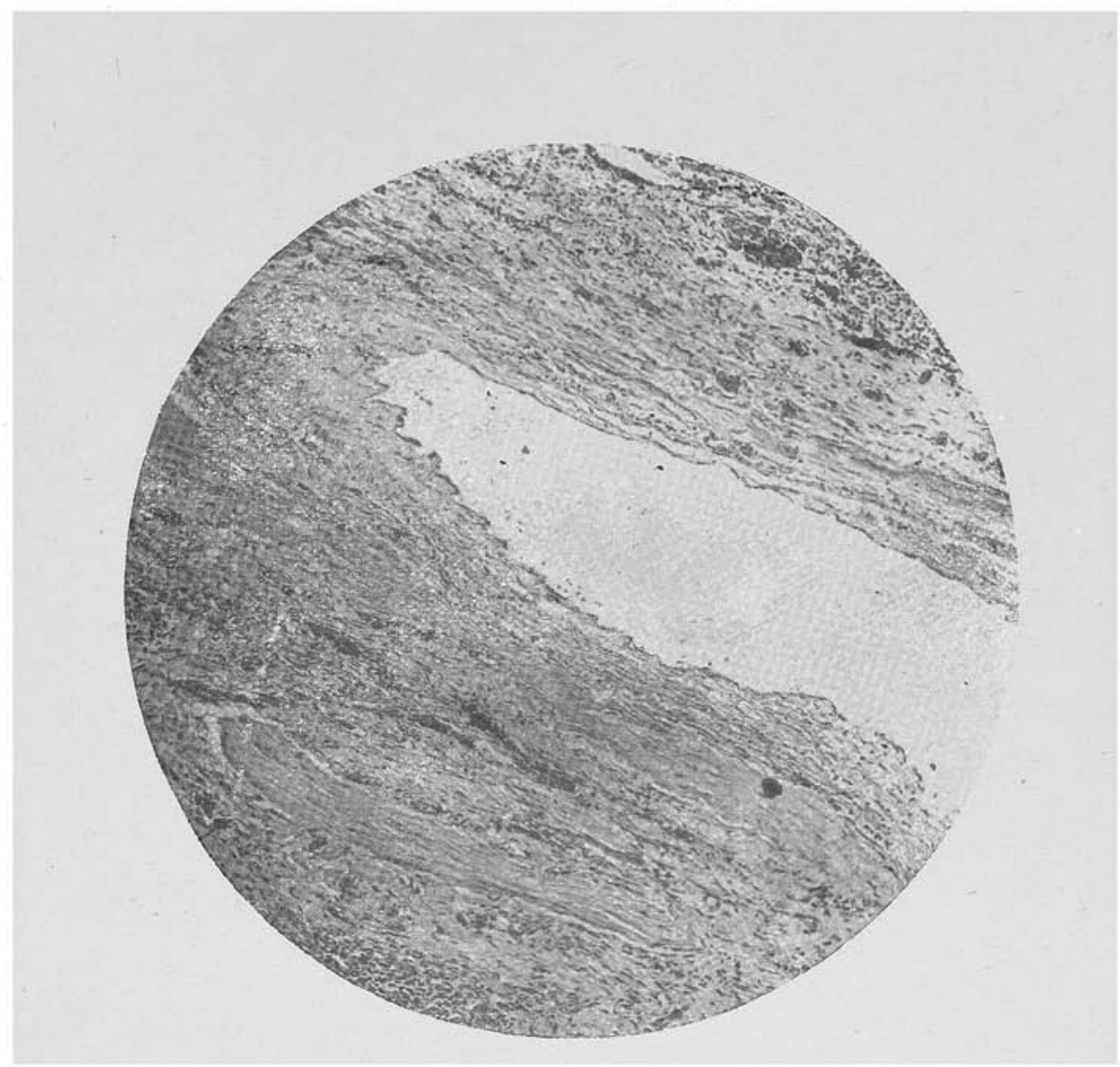

FIG. 6

Lymphangite obliterante peri-arterial 


\section{Estactistica dA FREQUENCIA RELATIVA OA Broncho preumonia Fibrosante post.grippal}

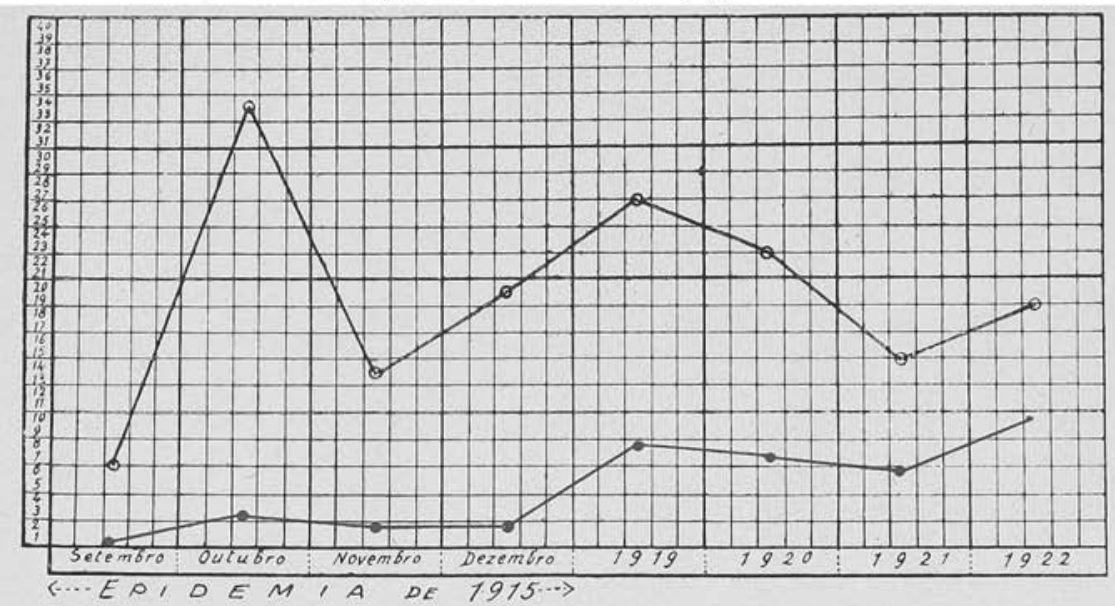

FIG. 7 


\section{ESTATISTICA FEITA COM OS PROTOCOLLOS DA ESCOLA DE MEDICINA DA UNIVERSIDADE DE YALE, NEW HAVEN, E. U. A.}

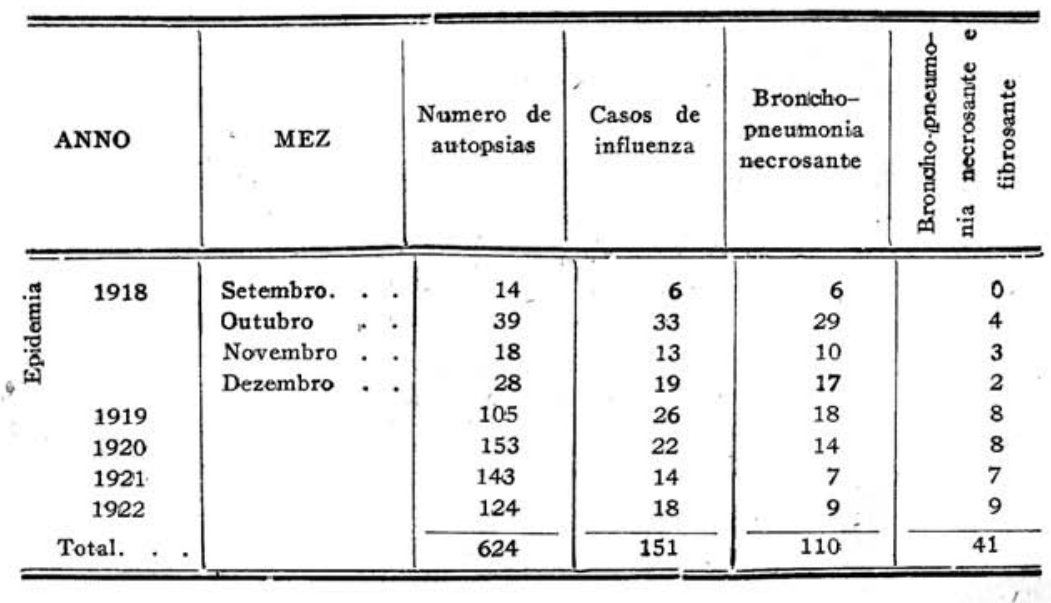

\section{PHYSIO-PATHOLOGIA}

Os disturbios funccionaes consequentes a esse processo vêm, como elle, gradual e progressivamente.

A' medida que a fibrose progride vae-se estabelecendo um obstaculo mecanico á corrente sanguinea na pequena circulação.

o coração tendo de vencer essa resistencia constante reagirá hypertrophiando-se, até que venha a dilatação e consequente insufficiencia.

A inflammação peri-bronchica destroe a resistencia das $\mathrm{pa}^{x}$ redes dos canaes, permittindo então que se dilatem pelos esforços da tosse.

Essas bronchliectasias que ensombream o prognostico são brazas sob cinzas que o menor vento pode reavivar, viveiro de germens cuja virulencia pode ser exaltada em dadas circumstancias.

$\mathrm{Si}$ as condições pulmonares furem compativeis com a vida, irá o doente cahindo em cachexia cardiaca progressive.

\section{APPLICAÇÕES CLINIOAS}

SYMPTOMATOLOGIA:

Os incommodos desses pacientes datam de um ataque severo de influenza epidemica de que foram victimas.

Dahi para cá contam elles como symptomas geraes insomnia mais ou menos rebelde e uma certa irritabilidade nervosa. 
Acham-se geralmente bem nutridos e conservam o appetite. A tosse é constante mas de intensidade variavel, indo desde a tosse ligeira até á mais rebelde, nada tendo, porém, de peculiar.

Escarram regularmente, ás vezes após uma crise paroxistica sobrevêm escarros abundantes, indicando esse facto a existencia de uma bronchiectesia.

E' possivel apparecerem filetes sanguineos no escarro, hemoptysès são excepcionaes.

Accusam dores thoraxicas que nada têm de caracteristico.

São communs dyspnéas, palpitações, tonturas e dôres precordiaes

Ao exame physico observam-se signaes variaveis.

A forma thoraxica as vezes se acha intacta, a expansão dos pulmões avaliada pela mobilidade do thorax está mais ou menos conservada; ha numerosos casos, porém, de adherencias pleuraes que the deformam a architectura. A percussão encontra a sonoridade normal ou pouco augmentada; na metade dos casos ha macicez nas bases.

A sonoridade dos apices é normal ou pode haver ligeira submacicez.

A respiração é geralmente rude e soprosa, e a expiração prolongada.

Habitualmente o fremito não apresenta modificações.

|Sibilos e estertores sonoros são communs na base, raramente alcançum os apices.

Nunca apparecem nos apices os estertores crepitantes e subcrepitantes typicos da tuberculose.

O exame radiologico demonstra um aspecto anormal mas o radiologista raramente firma um diagnostico.

Os relatorios falam em condensações diffusas, espessamentos peri-bronchicos, dilatações de toda a arvore bronchice, pequenas areas de densidade augmentada, infiltração das bases, condensações dos hilos, e aventam hypotheses de se tratar de bronchite, tuberculose broncho-pneumonica, tisica fibrosa, syphilis do pudmão, etc.

Esses casos que não mostram lesões dos apices requerem estudos cuidadosos para que se chegue a uma conclusão.

As injecções intra-tracheaes de lipiodol esclarecem sobre a presença de bronchiectasias.

A temperatura frequentemente é normal, ás veze $\mathbf{S}_{S}$ ha elevações vespertinas a $37^{\circ}, 2$.

O escarro nada tem de caracteristico. Não são encontrados B. de Koch, espiraes de Curschmann e crystaes de Charcot-Leyden, Ha abundantes cellulas de pús.

DIAGNOSTICO DIFFERENCIAL:

1) Tuberculose - $O$ curso da molestia simula a tuberculose. 
Ha, no emtanto, certas differenças. Os symptomas toxicos, se presentes, são os do syndromo cardiaco ou de irratibilidade nervosa.

As lesões não se localizam nos apices como na tuberculose; quando localisadas preferem as bases.

Os signaes radiologicos não são tão bem definidos como na tuberculose. A ausencia de bacillos de Koch no escarro verificada após exames repetidos, juntamente com a presença na historia clinica de uma grippe pulmonar severa e ausencia de estertores sub-crepitantes, são outros tantos indicios contrarios á bacillose.

Alguns autores modernos acham que é erronea a noção de que a grippe predispõe á tuberculose, encontrando nas confusões diagnosticas da fibrose post grippal o fundamento dessa noção.

2) Molestia do coração - Praticamente todos os pacientes exhibem phenomenos cardiopathicos. E' commum encontrarmos dyspnéa de esforço, palpitações, dôres precordiaes, e ás vezes sopros funcionaes.

Encontra-se um syndromo do coração direito que, isolado, deve levar o clinico ao exame dos pulmões, mórmente si o doente conta ter tido grippe.

Ha casos diagnosticados syphilis cardio-aortica. Nesses casos a segunda bulha parece um pouco prolongada e hypersonora, provavelmente devido ao rapido desdobramento da mesma.

A historia clinica, o Wassermann negativo e a falta de outros signaes afastam o diagnostico da syphilis cardiaca.

3) Hyperthyrodismo - A excitabilidade nervosa é por vezes tal que suggere o estudo do metabolismo basal, porém, a falta de exophtalmia, de bocio e de augmento do metabolismo são contrarios a esse diagnostico.

4) Asthma - o emphysema é ás vezes accentuado e existem ataques asthmatiformes. A oppressão é, porém, mais ou menos continua e não existem os typicos ataques com periodos remissivos intercalados. Não ha tambem susceptibilidade ás proteinas reactivas.

\section{DECURSO:}

A evolução é lenta e a morte sobrevem no fim de tempo mais ou menos dilatado.

A lesão é incuravel e o prognostico basea-se sobre a extensão da cirrhose, presença de bronchiectasias, estado geral e sobretudo estado funccional do coração.

\section{CONCLUSÕES}

1) A broncho-pneumonia grippal apresenta a particularidade de produzir mais frequentemente que as outras a fibrose pulmonar. 
2) Essa tendencia especial manifesta-se muito precocemente, em pleno periodo agudo, $\theta$

3) realiza a fibrose por um processo de alveolite e bronchiolite obliterantes.

4) A frequencia dos ıcasos de proliferação organizante é, na eutopsia, de $27,1 \%$.

5) Essa fibrose pulmonar crea obstaculo mecanico á pequena circulação, do qual se resente o coração diireito.

6) O diagnostico differencial é difficil, principalmente com a tuberculose.

7) Nos individuos que soffreram um insulto de grippe pulmonar severa, é necessario lembrar a possibilidade de uma fibrose post-grippal quando se cogite do diagnostico de tuberculose, e, utilisar perspicaz e judiciosamente todos os indicios para o estabelecimento de uma conclusão.

8) O prognostico dessa affecção é reservado.

\section{BIBLIOGRAPHA}

1) NOTHANAGEL - Specielle Pathologie und Therapie. - Vol. 4, 1." parte, pag. 106. Ediçăo: Vienna 1896

2) MC CRUDDEIN, F. H. - Post-influenzal ehronic pneumonitis. - J. Amer. Med. Assoc., 1923, vol. 80, pg. 609.

3) Fishatighr, M. - Pulmonary sequels of influenza. - M. Amer. J. of Medi. Sciences, - Philadellphia, 19121, vol. 161, pag. 365.

4) PIERSON, P. H. - Post-influenzas lung conditions. - Med. Clin. N. Am., - Phila., 1922-23, vol. 6, pag. 1509 .

5) NORIRIS AIND LAINDIS. - Diseases of the ehest. - Ed. 1920, pag. 462.

6) MAC CALLUU, W. G. - Pneumonia in army eamps. - Monograph of the Rockeffeller Institute, n. 1.0, Albril 16, 1919.

7) MAC CALLUM, W. G. - Pathology of the pneumonia following influenza. - J. Amer. Med. Assoic, 1919, vol. 72, pagr. 720.

8) WINTERnITZ, M. C. - Pathology of war gas poisoning -1920 .

9) WINTERNITZ, M. C. - Chronie lesions of the respiratory tract J Amer. Assi, 19119, vol. 73, pag. 689.

10) WAGNER, J. H. - Bronchiolitis obliterans following inhalation of acrid fumes.

11) KLOTZ, O. - Pathology of epidemie influenza, in studies on epidemie influenza, 1919, pag. 2017.

12) WINTERNITZ, SMITH AND MC NAMARA. - Epithelial proliferation following the intrabronchial insufflation of acid - $\mathrm{I}$.xp. Med., 1920. vol. 32, pg. 199. 